\title{
Clinical image: rare localization of a giant recurrent malignant meningioma
}

\author{
Ibrahim Halil Türkbeyler, Mehmet Akif Sarıca, Taner Babacan, İsmail Dilli \\ Department of Internal Medicine, Adiyaman University School of Medicine, Turkey
}

\begin{abstract}
Meningiomas are the most common benign brain tumors. Malignant meningioma is a rare subtype with a high recurrence rate and poor prognosis. Most meningiomas grow intracranially, whereas extracranial growth of meningiomas has been rarely reported. We herein report a rare case of a giant malignant meningioma with multiple recurrences. A 56-year old man presented with a forehead swelling. He had a history of repeated craniotomy for a frontal sinus invasion. The tumor recurred one year after the last surgery on the forehead and was classified as a giant meningioma at admission to our hospital. Neurosurgeons performed total resection of the giant meningioma and reconstructed the defect. However, six months after the surgery, the tumor recurred at the same site. The patient refused surgery and underwent cyber-knife treatment. An hydroxyurea treatment was initiated after radiotherapy, but the tumor progressed. Temozolomide and cisplatin treatment was started. A valuable clinical improvement was observed after the initiation of the first cycle. After completion of two cycles of systemic chemotherapy, the patient will be assessed clinically and radiologically.
\end{abstract}

\section{Case Report}

A 56-year old man with a history of recurrent frontal meningioma presented with a forehead swelling. Meningioma was originally diagnosed at age 53, when an enhancing frontal dura-based mass was detected on a magnetic resonance imaging (MRI) scan. The patient underwent near-total surgical resection followed by 34 fractions of adjuvant external-beam radiation therapy. Pathology showed an anaplastic-type meningioma with sheets of rhabdoid-like cells in focal areas of the tumor, geographic tumor necrosis, frontal bone destruction, dural invasion and increased mitotic activity. The patient underwent repeated craniotomy for three years

Correspondence: Ibrahim Halil Turkbeyler, Department of Internal Medicine, Adiyaman University School of Medicine, Adiyaman, Turkey.

Tel.: +90.532.7118366.

E-mail: turkbeyler@mynet.com

Key words: localization, malignant meningioma.

Received for publication: 19 October 2013.

Revision received: 13 December 2013.

Accepted for publication: 20 December 2013.

This work is licensed under a Creative Commons Attribution NonCommercial 3.0 License (CC BY-NC 3.0).

(C) Copyright I.H. Türkbeyler et al., 2014

Licensee PAGEPress, Italy

Italian Journal of Medicine 2014; 8:132-134

doi:10.4081/itjm.2014.438 to treat a recurrent frontal sinus anaplastic meningioma. The tumor recurred on his forehead one year after the last surgery and was classified as a giant meningioma on admission to our hospital. On physical examination, the patient's nervous system was not impaired, except for the mass on the frontal region, and no eyesight damage or abnormal reflections was found. The protruding mass measured approximately 6-4 cm, with uneven edges (Figure 1). To examine the lesion in more detail and to devise surgical strategies, MRI was performed. The MRI showed that the lesion was located in the bilateral frontal lobes, was regularly shaped, lobulated, invaded the skull, and was growing subcutaneously in the frontal sinuses of the skull (Figure 2). Considering the history of the patient and the results of the examinations, he was diagnosed with recurrent malignant meningioma of the frontal part of the skull. Neurosurgeons performed a total resection of the giant meningioma and reconstructed the defect with artificial dura and a local rotating flap. Histological examination revealed grade 3 anaplastic meningioma. The patient was lost at follow up. Six months after surgery, the tumor recurred at the same site. The patient refused surgery and underwent cyber-knife treatment. An hydroxyurea treatment with $15 \mathrm{mg} / \mathrm{kg}$ per day was initiated after the radiotherapy, but the tumor progressed. Due to the enormity of the recurrent mass, systemic temozolomide and cisplatin chemotherapy treatment was initiated. A valuable clinical improvement was observed after the initiation of the first cycle. After completion of two cycles of systemic chemotherapy, the patient will be assessed clinically and radiologically. 


\section{Discussion}

Meningiomas are usually described as benign tumors of the central nervous system. However, the anaplastic meningioma subtype is rare and malignant. Meningiomas can arise anywhere from the dura, most commonly within the skull and at sites of dural reflec- tion. Other less common sites include the optic nerve sheath and the choroid plexus. Approximately 10\% arise in the spine. ${ }^{1}$ Very rarely, meningiomas can occur at extradural sites. Primary extradural meningiomas represent about $2 \%$ of all meningiomas. ${ }^{2}$ The overall median time to recurrence is about three years. When they occur extracranially, they are typically treated by
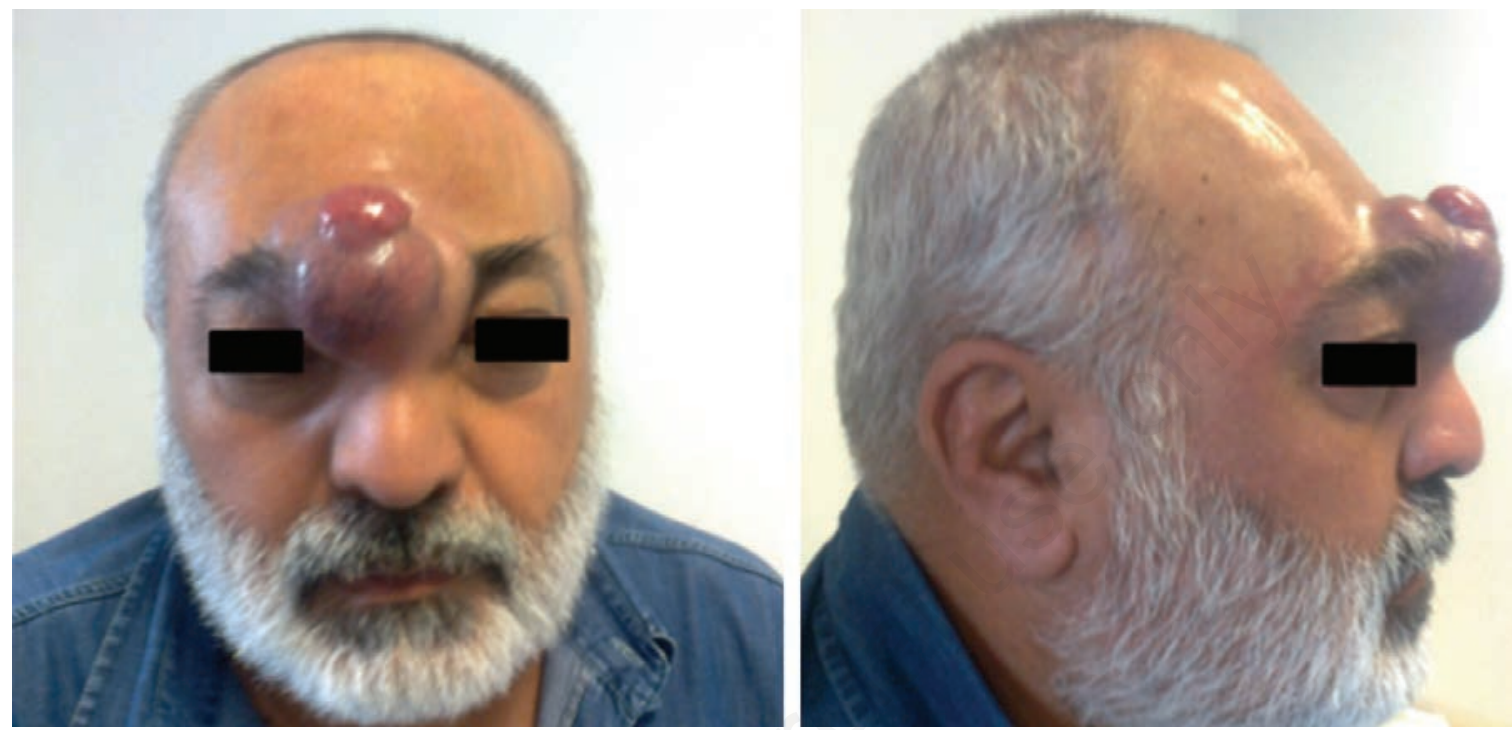

Figure 1. The protruding mass measured approximately 6-4 cm, with uneven edges.
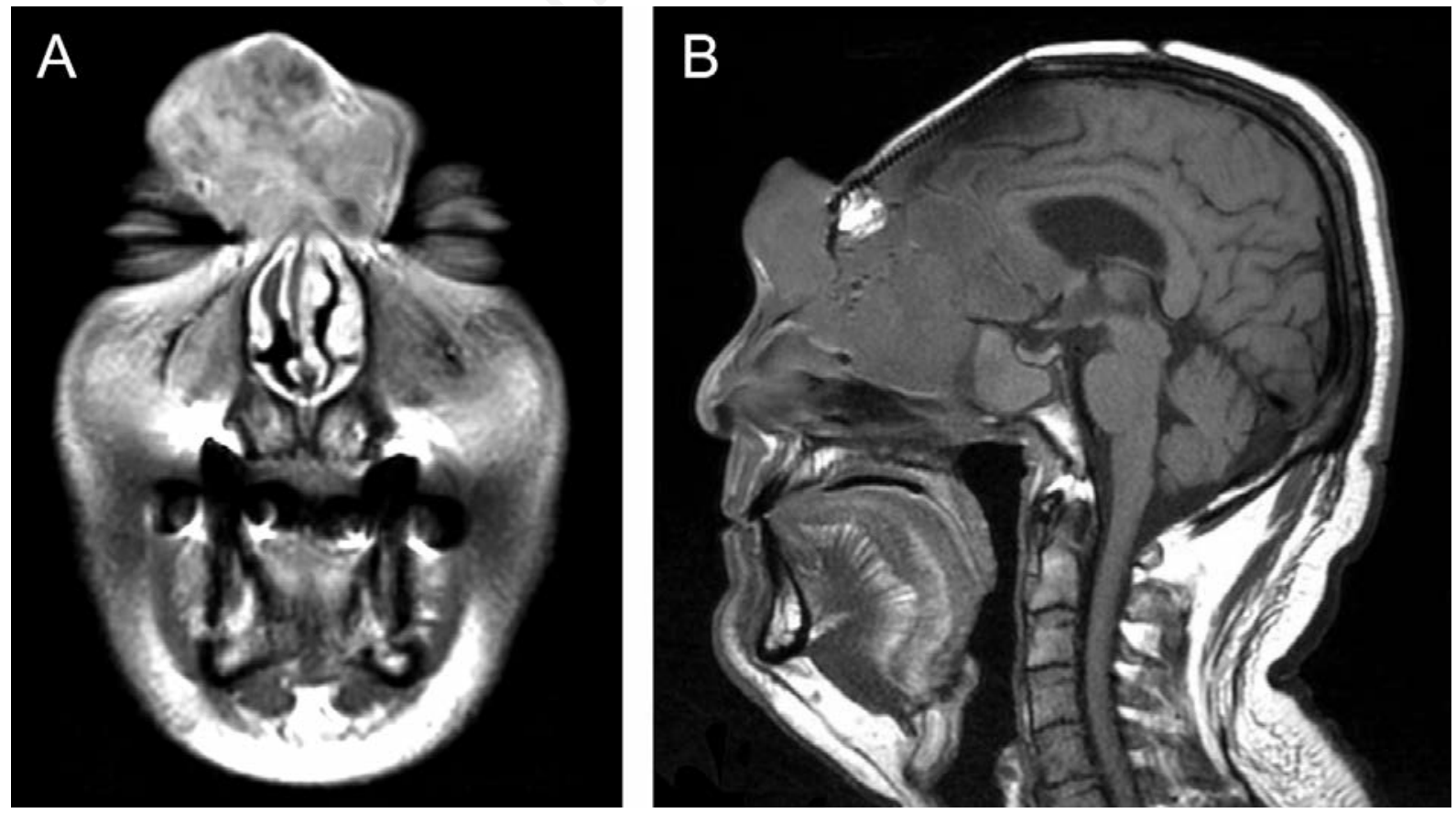

Figure 2. A and B) Magnetic resonance imaging showed that the lesion was located in the bilateral frontal lobes, was regularly shaped, lobulated, invaded the skull, and was growing subcutaneously in the frontal sinuses of the skull. 
surgeons unfamiliar with this tumor type. ${ }^{3}$ The clinical and radiographic presentation of benign, atypical, and malignant primary extradural meningiomas may be similar. Therefore, all types of meningiomas demand careful operative planning and postoperative treatment, including radiotherapy, chemotherapy and antiangiogenic treatment.

\section{References}

1. Whittle IR, Smith C, Navoo P, et al. Meningiomas. Lancet 2004;363:1535.

2. Figueroa BE, Quint DJ, McKeever PE, et al. Extracranial metastatic meningioma. Br J Radiol 1999;72:513-6.

3. Mattox A, Hughes B, Oleson J, et al. Treatment recommendations for primary extradural meningiomas. Cancer 2011;117:24. 